\title{
Functional Disorders and Comorbidity
}

By Jack M. Gorman, MD

It seems obvious to most people that the brain and the rest of the body interact. Stress seems to worsen most general medical conditions, mortality is higher among widowers than married men, and almost everyone becomes moody when he or she has a viral infection. However, these are mostly anecdotal observations. The precise ways in which mind and body relate in health and disease is more elusive and, at times, controversial.

Perhaps the hardest evidence we have for a mindbody connection comes from studies showing that depression is an independent-and, surprisingly, quite a robust-risk factor for cardiovascular disease. Following acute coronary syndrome depression significantly worsens outcome, including increasing all-cause mortality rates.

Another well-documented finding, going in the opposite direction, is the high rate of depression experienced by those receiving $\alpha$-interferon for the treatment of hepatitis $\mathrm{C}$ or malignant melanoma. Less secure is whether depression increases risk or worsens prognosis for cancer; why patients with schizophrenia are at greater risk for heart disease and cancer than the general population; if it is true that stress plays a role in exacerbations of multiple sclerosis; and what is the relationship between depression and the risk for Alzheimer's disease. In each there are conflicting epidemiological data and difficulties understanding the neural mechanisms that would broker the relationship between brain and targeted peripheral organ.

Even more controversial are a group of so-called functional disorders, including chronic fatigue syndrome, fibromyalgia, and, the subject of this month's issue of CNS Spectrums, irritable bowel syndrome (IBS). Most of these are diagnoses of exclusion, given only when the history, physical examination, laboratory tests, and $\mathrm{X}$-rays fail to reveal alternative disease. There are disputed claims that these functional syndromes are really only manifestations of underlying psychiatric illness, including depression and anxiety disorders. This view is supported by epidemiological studies that persistently find high rates of comorbidity between them and psychiatric illness and by the frequent recommendation to treat functional syndromes with antidepressant medications. I personally find neither of these convincing because depression and anxiety disorders are comorbid with almost all general medical conditions, including diabetes, heart disease, and cancer, and because antidepressants are known to have widespread effects outside of the central nervous system, including opposing platelet aggregation and affecting heart rhythm. Hence, whether conditions like IBS are legitimate independent diagnoses or form frustes for psychiatric illness remains unanswered.

What is clear and also elegantly put forward by the articles assembled for this month's CNS Spectrums by $\mathrm{R}$. Bruce Lydiard, MD, PhD, is that IBS is a common and extremely disabling condition that is often accompanied by depression or an anxiety disorder, such as panic disorder. While there is evidence that antidepressants may be helpful, my take on the data presented in these articles is that studies evaluating them for IBS are mixed and that cognitive-behavioral therapy may give more consistent positive results.

Just how is the brain connected to the gastrointestinal (GI) tract? I will leave the definitive answer to a question like that to people such as my daughter, Rachel Gorman, who has just entered medical school and is currently enduring a rite of passage to a medical career: gross anatomy. She and her colleagues in first-year medical school classes probably know better than I which nerves connect to which parts of the GI tract. But as we learned from Michael Gershon, MD, in his wonderful book, The Second Brain, there are more serotonergic neurons in the GI tract than in the brain. Interestingly, however, Dr. Gershon tells us that there is no direct connection between serotonin neurons in the brain and the gut. Hence, if antidepressants do work for IBS, it is entirely possible that they do so by virtue of a direct effect on neurons in the GI tract rather than because of top-down effects from the brain.

Most of us have experienced abdominal discomfort during unpleasant events and felt nauseous when presented with disgusting material. Patients with anxiety and depression often complain of GI distress and patients with GI disease of known pathology, like ulcer or inflammatory bowel disease frequently suffer from mood and anxiety disorders. It seems evident that there must be a connection between mind and gut in there somewhere. Hopefully, someone, perhaps a first-year medical school student, whose entire existence is now consumed with what goes where in the human body, will help ort this out one day. CNS

Dr. Gorman is the editor of this journal and professor of psychiatry and professor of neuroscience in the Department of Psychiatry at Mount Sinai School of Medicine in New York City. 